\title{
LABORATORY TESTING OF VELOCITY SENSING IN A MAGNETORHEOLOGICAL DAMPER WITH POWER GENERATION
}

\author{
Bogdan SAPIŃSKI \\ Department of Process Control, AGH University of Science and Technology, aleja Mickiewicza 30, 30-059 Cracow, Poland \\ deep@agh.edu.pl
}

received 15 February 2017, revised 18 July 2017, accepted 7 August 2017

\begin{abstract}
The study summarises the results of experimental examination of velocity sensing capability in a prototype of a magnetorheological damper with power generation (MRD). The device has two main components: an electromagnetic power generator and an MR damper. The study outlines the structure of the device with the main focus on the generator part, and provides results of tests performed under the idle run. The discussion of demonstrates the potentials of MRD action as a velocity-sign sensor and presents key issues which need to be addressed to enable its real life applications.
\end{abstract}

Key words: MR Damper, Power Generator, Voltage, Velocity, Sensing

\section{INTRODUCTION}

Recent years have witnessed a major advances in selfpowered MR dampers which are able to recover energy from external excitations and to adjust themselves to excitations by varying damping characteristics. It has also been established that information about relative velocity across such MR dampers can be extracted on voltage generated by the generator giving the devices the self-sensing capability i.e. can act as a velocity sensors. An increasing number of research reports on this subject have appeared in the last decade. For example, (Jung et al., 2009) investigated experimentally the sensing capability of the generator incorporated in the MR damper-based vibration control system and demonstrated that the device may be considered as a velocity-sign sensor. Also, (Jung et al., 2010) studying a sensing capability of such system showed that the generator could act as a velocity sensor for common control methods in MR damperbased systems. (Wang et al., 2010) proposed an integrated relative displacement self-sensing MR damper to realize the integrated relative displacement sensing and controllable damping. Based on the extension of the presented idea, the prototype of such MR damper was designed, fabricated and tested by (Wang and Bai, 2013). (Chen and Liao, 2010) reported that it is possible to engineer an MR damper with the power generation feature which integrates energy harvesting, dynamic sensing and MR damping technologies in a single device. Also, (Chen and Liao, 2012) performed theoretical and experimental studies of an MR damper prototype which had both self-powered and self-sensing capability. (Zhu et al., 2012) presented self-powered and sensor-based MR damper systems since such systems could be particularly useful in large-scale civil constructions where the power supply is impractical. (Li et al., 2013a, 2013b) presented an innovative concept of a mechanical motion rectifier which converts bidirectional motion into unidirectional motion.

This paper deals with velocity sensing of the MRD prototype and recalls three former works of the author (Sapiński, 2011, 2014, Sapiński et al., 2016)). The study is organized as follows. Section 2 describes the structure of the device with the main focus on the power generator part. Section 3 provides results of tests performed under idle run to demonstrate the self-sensing potentials. The conclusions are given in Section 4.

\section{STRUCTURE OF THE DEVICE}

The sectional view of the MRD with numeric symbols (1-17) indicating its crucial components is shown in Fig. 1. Two main components are the electromagnetic power generator (harvester) and the MR damper. The generator is axially symmetrical and complete with three systems of permanent magnets (three magnets in each), two inner spacers (height $9 \mathrm{~mm}$ ) and two outer spacers (height $6.5 \mathrm{~mm}$ ), and the coil (height $18.5 \mathrm{~mm}$, inside diameter $31 \mathrm{~mm}$, outside diameter $77 \mathrm{~mm}$, distance between coil sections $5 \mathrm{~mm}$ ) with two winding sections incorporating 273 turns each, wound on a carcass (height $20.5 \mathrm{~mm}$, wall thickness $1 \mathrm{~mm}$ ) with copper foil with one-sided insulation (foil thickness -0.05 $\mathrm{mm}$, insulation thickness $-0.03 \mathrm{~mm}$ ). The magnets made of $\mathrm{NdFeB}$ grade $\mathrm{N} 35$, are ring-shaped (inside diameter $12 \mathrm{~mm}$, outside diameter $30 \mathrm{~mm}$, height $5 \mathrm{~mm}$ ) and display axial magnetisation with direction indicated by arrows. The magnets and spacers are mounted on a non-magnetic stainless steel shaft. Coil winding sections, placed inside a housing (height $50 \mathrm{~mm}$, wall thickness $3 \mathrm{~mm}$ ), are connected such that as the moving part moves with surrounding vibration, they experience a change in flux linkage and thus induced voltages should sum up. The coil housing and spacers are made of ferromagnetic steel SAE 1215. The generator components are placed inside the housing, locked with the lower and upper covers. Sleeves provided in the cover ensure the axial movements of the piston rod. The fixture to hold the generator is attached to the lower cover. The height of an air slit between the carcass and the magnet systems is $0.5 \mathrm{~mm}$. 


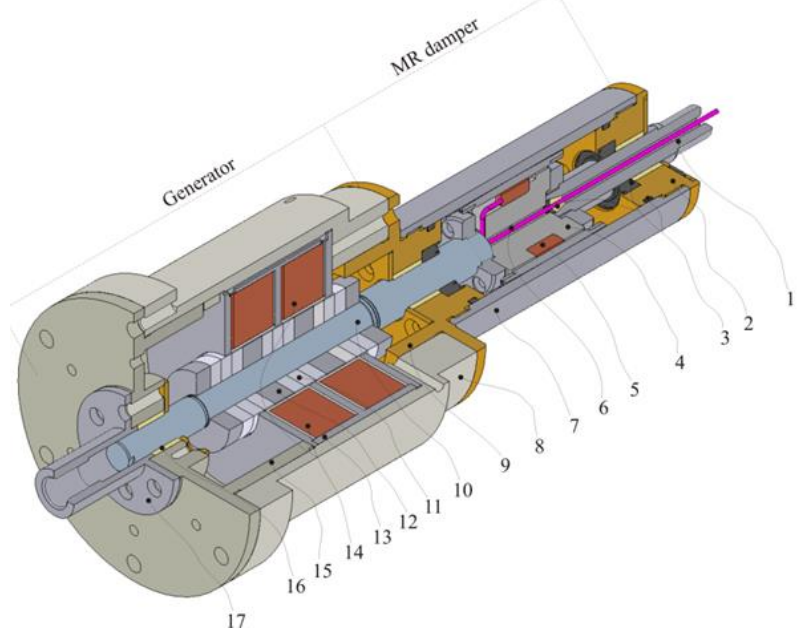

Fig. 1. Sectional view of the MRD: 1 - piston rod, 2 - brass lid, 3 - rubber seals, 4 - piston, 5 - control coil, 6 - wire, 7 - damper housing, 8 - device housing, 9 - brass connector, 10 - rod, 11 - neodymium magnets, 12 - spacers, 13 - housing, 14 - coils, 15 - lid, 16 - slip sleeves, 17 - holder

The damper is an axially-symmetrical single-tube damper with a circumferential slit. The piston rod made of non-magnetic austenite stainless steel is attached to the piston via ferromagnetic kidney plates and sealed with rings. The rings together with the sleeves are placed in two co-axial guides to guide the piston rod. The damper coil is made of copper wire $0.4 \mathrm{~mm}$ in diameter wound on the carcass, forming 306 windings. The coil is placed on the core made of ferromagnetic steel SAE 1215. The lead power-supplying the control coil is let out via a hole in the piston rod. The damper housing, made of ferromagnetic steel SAE 1025, is locked on both ends by covers made of austenitic stainless steel. The damper contains $36.5 \mathrm{ml}$ of the MR fluid. The resistance of the generator coils is $141 \mathrm{mH}$ and the resistance is $2.45 \Omega$. The geometric parameters of the device are summarised in (Sapiński, 2014).

\section{TESTS, RESULTS AND DISCUSSION}

The MRD was tested in the test rig (see Fig. 2) that incorporates the MTS test machine (www.mts.com), the data acquisition system comprising a portable computer, I/O board DAQPad6052E of National Instruments and the supporting software LabView. The MTS machine was programmed to generate sine and triangular excitations with an amplitude of $0.01 \mathrm{~m}$ and a frequency of $1 \mathrm{~Hz}$ and $6 \mathrm{~Hz}$. Tests were carried out during the idle run of the MRD generator part. The measurements of output voltage (electromotive force) $u$ were taken and position $x$ was duly registered. The values of $u$ and $x$ were recorded in 10 cycles of the piston motion. The sampling frequency for each channel was $1 \mathrm{kHz}$ per cycle. The piston velocity $\dot{x}$ was obtained by differentiating the position $x$.

The results revealed the velocity sensing capability of the MRD, demonstrated by exemplary plots shown in Figs 3-4 showing the time histories (relative time $t \cdot f$ ) of piston velocity $\dot{x}$ and voltage $u$ induced in the generator under sine excitations with amplitude of $0.01 \mathrm{~m}$ and frequency of 1,3 and $6 \mathrm{~Hz}$. It is apparent that plots in Fig. 4 agree with the plots in Fig. 3, though there are certain discrepancies. Plots of velocity $\dot{x}$ plots follow the sine wave patterns whilst of voltage $u$ are shaped like deformed sine lines.
These deformations are related to configuration of the magnetic circuit in the generator (the system of magnets) and the associated flux density distribution. Plots reveal characteristic peaks, resulting from the changes of the piston velocity sign. It appears that velocity plots are not smooth, which is caused by the fact the velocity signal is obtained via numerical differentiation of the registered position signal $x$, which involves noise. This issue can be illustrated by comparing the graphs in Fig. 5, plotting the differentiated signal of an ideal sine wave and that produced by the test machine, registered as a sine wave (excitation) $x$ and then differentiated. It is worthwhile to mention that velocity and voltage plots pass through the zero point at the identical time instants.

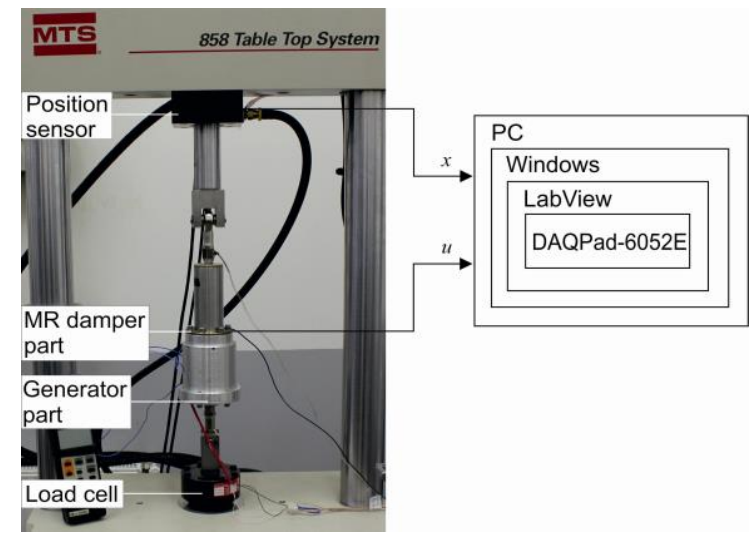

Fig. 2. Schematic diagram of the test rig

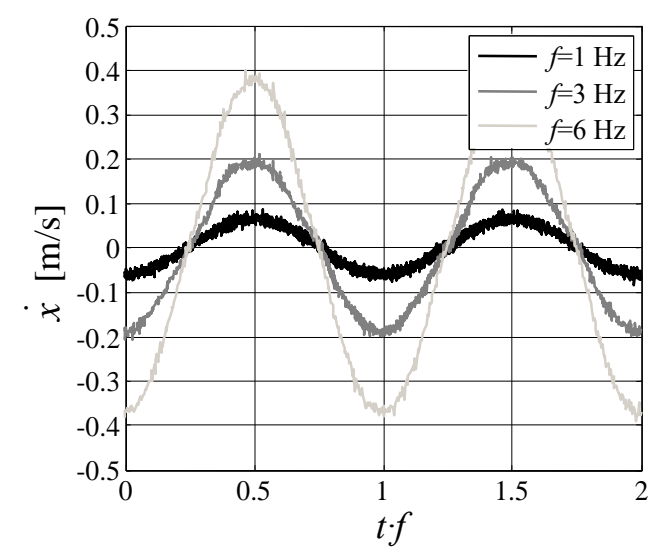

Fig. 3. Time histories of velocity $\dot{x}$; sine excitation, amplitude $0.01 \mathrm{~mm}$

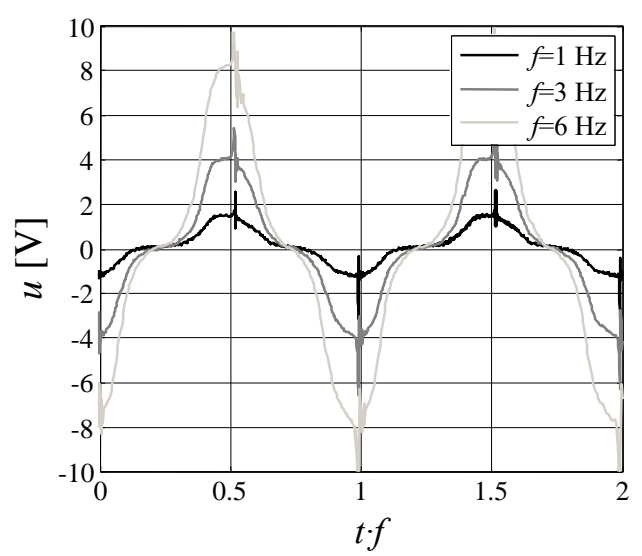

Fig. 4. Time histories of voltage $u$; sine excitation, amplitude $0.01 \mathrm{~mm}$ 


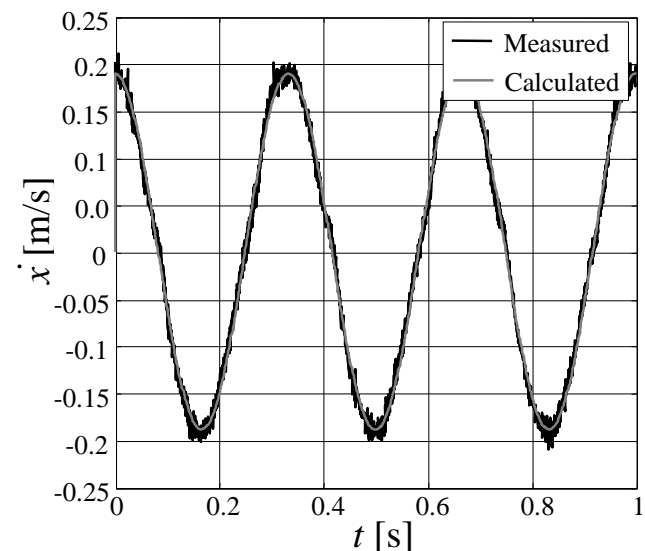

Fig. 5. Time histories of velocity after differentiating and calculating $\dot{x}$; sine excitation, amplitude $0.01 \mathrm{~m}$, frequency $3 \mathrm{~Hz}$

It should be noticed that for an ordinary electromagnetic power generator the permanent magnets generate the magnetic field whose distribution in the generator's region is determined by configuration of magnets and their position with respect to the coils' housing. The magnets remaining in respective movement with regard to the coil give rise to induction of voltage in the coil, that voltage being proportional to the velocity. In consideration of the fact that velocity of magnets is low, the effects of eddy currents induced in conducting elements of the generator can be neglected. Then the output voltage is proportional to the velocity. However, for the specially designed power generators the relationship between these two quantities is not always straightforward. Therefore, to obtain the direct information about velocity, special velocity sensing algorithms have to be developed.

In the work (Sapiński et al., 2016) only the self-powered capability of the MRD was tested. The main objective of the tests performed in this study was to confirm that the MRD could provide information about velocity across the damper that could be useful for controlling system dynamics. This can be achieved by implementation of control algorithms that utilize the velocity across the damper to determine control output (Karnopp et al., 1974; Jansen and Dyke, 2000). As demonstrated in (Chen and Liao, 2012) such approach requires an appropriate sensing function. For this purpose the authors proposed and validated a velocity-sensing method for the developed self-sensing MR damper with power generation. The method, however, requires real-time signal processing. The advantage of the defined sensing function is that it can be applicable to various control algorithms. Moreover the developed device will have good performance for broad vibrationdamping applications.

As described in Section 2, the MRD has the damper part and the generator part. The damper part, having a piston assembly, is moveable to the cylinder under an external excitation while the generator part produces electrical power according to the relative movement between the piston and the cylinder assembly. On the basis of the acquired $u$ and calculated $\dot{x}$ data sets registered at sine and triangular excitations with an amplitude of $0.01 \mathrm{~m}$ and a frequency of $1 \mathrm{~Hz}$ and $6 \mathrm{~Hz}$, the relationship between these two quantities was identified. In this procedure the function polyfit.m available in MATLAB was used. Let us assume that that the relationship between e andx can be approximated by the following polynomial of 1-st order $u=\kappa \dot{x}+\sigma$. If so, the value of directional coefficient kand shift coefficient $\sigma$ have to be calculated. Fig. 6 compares the plot of rms $u$ versusxiobtained from experi- mental data with that calculated in the identification procedure. The plot denoted by dash line was derived on the calculated coefficients $\kappa \mathrm{f}=21.86 \mathrm{~V} \cdot \mathrm{s} / \mathrm{m}$ and $\sigma=0 \mathrm{~V}$. It can be seen that $\mathrm{rms} u$ as function $\dot{x}$ is nearly linear. Time histories of velocity $\dot{x}$ and $u=21.86 \dot{\mathrm{x}}$ obtained under sine excitation at amplitude of 0.01 $m$ and frequency of 1 and $4 \mathrm{~Hz}$ are shown in Fig. 7 .

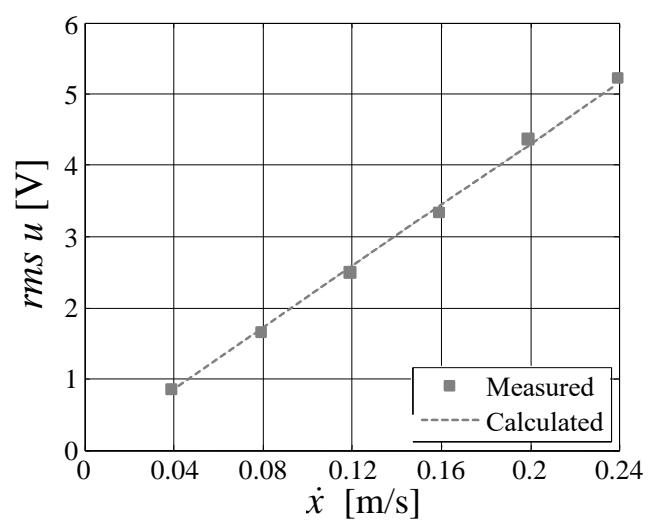

Fig. 6. Voltage $r m s u$ vs. velocity $\dot{x}$
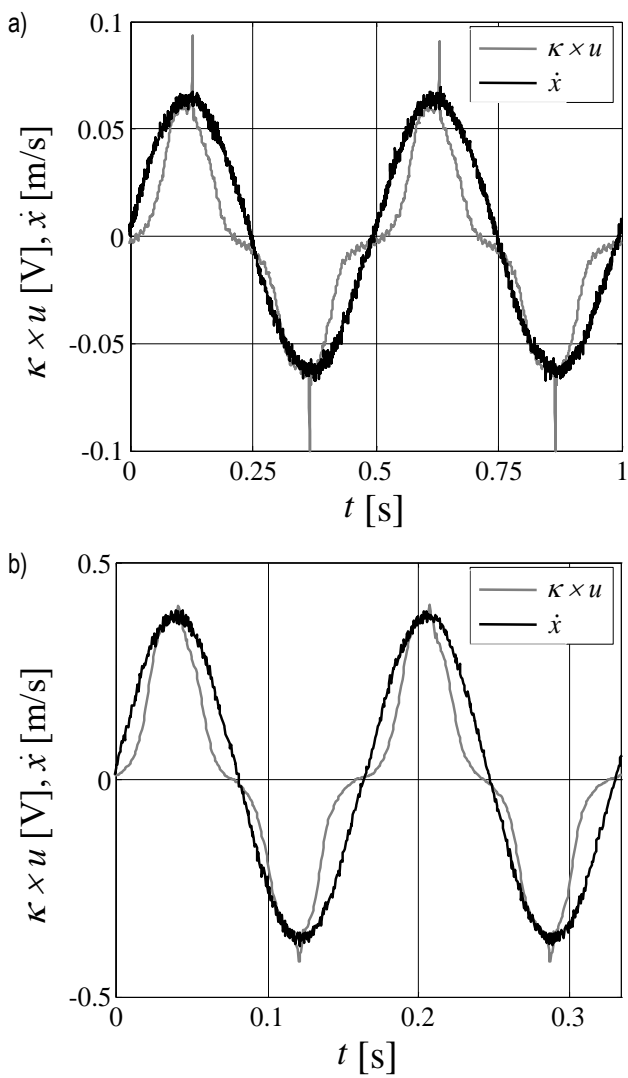

Fig. 7. Time histories of velocity $\dot{x}$ and voltage $u$; sine excitation, amplitude $0.01 \mathrm{~mm}$, frequency: a) $f=1 \mathrm{~Hz}, \mathrm{~b}) f=4 \mathrm{~Hz}$

\section{SUMMARY}

The study investigates the velocity sensing capability in the MRD prototype. For this purpose the generator part of the device was tested under the idle run in laboratory conditions. The measurements of output voltage of the generator and position were 
taken and the tests results were processed accordingly. The obtained time histories of voltage and velocity were then analysed. The linear relationship between voltage and velocity was established enabling the comparison of registered voltage and velocity signals reconstructed from voltage data sets. The discussion of test results reveals that in order to reconstruct the velocity signal from the voltage produced by the generator a special velocity-sensing method involving real-time signal processing has to be developed. The tested velocity sensing capability of the MRD is most useful for collecting dynamic information required for system dynamics control.

Further research efforts will concentrate on the method development and its validation at higher excitation frequencies and on reconstruction of velocity signals from voltage produced by applying an accelerometer instead of the position sensor.

\section{REFERENCES}

1. Chen C., Liao W. H. (2010), A self-powered, self-sensing magnetorheological damper, Proceedings of IEEE Conference on Mechatronics and Automation, 1364-1369.

2. Chen C., Liao W. H. (2012), A self-sensing magnetorheological damper with power generation, Smart Materials and Structures, 21, 025014.

3. Jansen L. M., Dyke S. J. (2000), Semi-active control strategies for MR dampers. ASCE, Journal of Engineering Mechanics, 126(8), 795-803.

4. Jung H. J., Jang D. D., Cho S. W., Koo J. H. (2009), Experimental verification of sensing capability of an electromagnetic induction system for an MR fluid damper based control system, 11th Conference on Electrorheological Fluids and Magnetorheological Suspensions, Journal of Physics: Conference Series, 149, 012058.

5. Jung H. J., Jang D. D., Koo J. H., Cho S. W. (2010), Experimental Evaluation of a 'Self-Sensing Capability of an Electromagnetic Induction System Designed for MR Dampers, Journal of Intelligent Material Systems and Structures, 21, 837-836.

6. Karnopp D. C., Crosby M. J., Harwood R. A. (1974), Vibration control using semi-active force generator, ASME Journal of Engineering for Industry, 96(2), 619-626.
7. Li Z, Zhuo L, Luhrs G, Lin L., Qin Y.( 2013b), Electromagnetic Energy harvesting shock absorbers: design, modeling and road tests, IEEE Transactions Vehicle Technology, 62, 1065-74.

8. Li Z., Zhuo L., Kuang J., Luhrs G. (2013a), Energy-Harvesting Shock Absorber with a Mechanical Motion Rectifier, Smart Materials and Structures, 22, 028008.

9. Sapiński B. (2011), Experimental study of a self-powered and sensing MR damper-based vibration control system, Smart Materials and Structures, 20, 105007.

10. Sapinski B. (2014), Energy harvesting MR linear damper: prototyping and testing, Smart Materials and Structures, 23, 035021.

11. Sapinski B., Rosół M., Węgrzynowski M. (2016), Investigation of an energy harvesting MR damper in a vibration control system, Smart Materials and Structures, 25, 125017.

12. Wang D. H., Bai X. X. (2013), A magnetorheological damper with an integrated self-powered displacement sensor, Smart Materials and Structures, 22, 075001.

13. Wang D. H., Bai X. X., Liao W. H. (2010), An integrated relative displacement self-sensing magnetorheological damper: prototyping and testing, Smart Materials and Structures, 19, 105008.

14. Zhu S. Y., Shen W. A., Xu Y. L., Lee W. C. (2012), Linear electromagnetic devices for vibration damping and energy harvesting: Modeling and testing, Engineering Structures, 34, 198-212.

15. www.mts.com

This work is supported by AGH University od Science and Technology under research program No. 15.11.130.431. 